\title{
Myeloperoxidase oxidation of methionine associates with early cystic fibrosis lung disease
}

\author{
Joshua D. Chandler ${ }^{1,2,3}$, Camilla Margaroli ${ }^{1,2}$, Hamed Horati ${ }^{4}$, \\ Matthew B. Kilgore ${ }^{1,2}$, Mieke Veltman ${ }^{4}$, H. Ken Liu ${ }^{3}$, Alexander J. Taurone ${ }^{5}$, \\ Limin Peng ${ }^{5}$, Lokesh Guglani ${ }^{1,2}$, Karan Uppal ${ }^{3}$, Young-Mi Go ${ }^{3}$, \\ Harm A.W.M. Tiddens ${ }^{4}$, Bob J. Scholte ${ }^{4}$, Rabindra Tirouvanziam ${ }^{1,2,6}$, \\ Dean P. Jones ${ }^{3,6}$ and Hettie M. Janssens ${ }^{4,6}$
}

\begin{abstract}
Affiliations: ${ }^{1}$ Center for CF and Airways Disease Research, Children's Healthcare of Atlanta, Atlanta, GA, USA ${ }^{2}$ Division of Pulmonary, Allergy and Immunology, Cystic Fibrosis and Sleep Medicine, Dept of Pediatrics, Emory University, Atlanta, GA, USA. ${ }^{3}$ Division of Pulmonary, Allergy and Critical Care Medicine, Dept of Medicine, Emory University, Atlanta, GA, USA. ${ }^{4}$ Division of Respiratory Medicine and Allergology, Dept of Pediatrics, University Medical Center Rotterdam, Erasmus MC-Sophia, Rotterdam, The Netherlands. ${ }^{5}$ Dept of Biostatistics, Emory University School of Public Health, Atlanta, GA, USA. ${ }^{6}$ These authors are joint senior authors.
\end{abstract}

Correspondence: Rabindra Tirouvanziam, Emory Children's Center, 2015 Uppergate Drive NE, Room 344, Atlanta, GA 30322, USA. E-mail: tirouvanziamdemory.edu

@ERSpublications

Identifying molecules associated with early CF lung disease may lead to new means of limiting progression. We found that airway fluid methionine sulfoxide produced by myeloperoxidase associates with lung disease in CF patients aged 1-3 years. http://ow.ly/m0u630lnSx3

Cite this article as: Chandler JD, Margaroli C, Horati H, et al. Myeloperoxidase oxidation of methionine associates with early cystic fibrosis lung disease. Eur Respir J 2018; 52: 1801118 [https://doi.org/10.1183/ 13993003.01118-2018].

ABSTRACT Cystic fibrosis (CF) lung disease progressively worsens from infancy to adulthood. Diseasedriven changes in early CF airway fluid metabolites may identify therapeutic targets to curb progression.

CF patients aged 12-38 months ( $n=24$; three out of 24 later denoted as CF screen positive, inconclusive diagnosis) received chest computed tomography scans, scored by the Perth-Rotterdam Annotated Grid Morphometric Analysis for CF (PRAGMA-CF) method to quantify total lung disease (PRAGMA-\%Dis) and components such as bronchiectasis (PRAGMA-\%Bx). Small molecules in bronchoalveolar lavage fluid (BALF) were measured with high-resolution accurate-mass metabolomics. Myeloperoxidase (MPO) was quantified by ELISA and activity assays.

Increased PRAGMA-\%Dis was driven by bronchiectasis and correlated with airway neutrophils. PRAGMA$\%$ Dis correlated with 104 metabolomic features $(\mathrm{p}<0.05, \mathrm{q}<0.25)$. The most significant annotated feature was methionine sulfoxide (MetO), a product of methionine oxidation by MPO-derived oxidants. We confirmed the identity of MetO in BALF and used reference calibration to confirm correlation with PRAGMA-\%Dis (Spearman's $\rho=0.582, p=0.0029$ ), extending to bronchiectasis (PRAGMA-\%Bx; $\rho=0.698, p=1.5 \times 10^{-4}$ ), airway neutrophils $(\rho=0.569, p=0.0046)$ and BALF MPO $\left(\rho=0.803, p=3.9 \times 10^{-6}\right)$.

BALF MetO associates with structural lung damage, airway neutrophils and MPO in early CF. Further studies are needed to establish whether methionine oxidation directly contributes to early CF lung disease and explore potential therapeutic targets indicated by these findings. 


\section{Introduction}

Cystic fibrosis (CF) is a multiorgan disease caused by genetic mutations affecting expression, stability, regulation and/or function of the $\mathrm{CF}$ transmembrane conductance regulator (CFTR) anion channel protein. $\mathrm{CF}$ is characterised by progressive bronchiectasis resulting in respiratory failure, the primary cause of mortality [1]. During childhood, CF airways exhibit neutrophilic inflammation, infections and impaired mucociliary clearance [2]. Inflammation may be key to lung disease development due to its early onset, before routine detection of pathogens. Indeed, presence of neutrophil elastase in airway fluid was the strongest predictor of development of persistent bronchiectasis in a study of young children with CF [3]. Neutrophil release of primary granules, which contain neutrophil elastase, myeloperoxidase (MPO) and other proteins capable of injuring airway mucosa, is a plausible mechanism of CF airway disease pathogenesis [4]. Recognising the earliest onset of inflammation in CF and underlying mechanisms is key to limiting disease progression.

Airway lining fluid, chiefly regulated by epithelia, could play major roles in promoting CF airway inflammation $[5,6]$. CFTR regulates airway fluid composition and volume directly and through interaction with other proteins, such as the epithelial sodium channel [1]. When CFTR function is insufficient, airway fluid may change in ways that promote disease, either providing direct pro-inflammatory cues or injuring airway cells [7]. Incoming neutrophils, via intense metabolic and effector activities, may further alter CF airway fluid. Due to the complexity of human airway physiology, unbiased methodologies such as untargeted metabolomics may serve to identify unanticipated biochemical changes driving pathogenesis.

Sensitive chest computed tomography (CT) techniques have been developed to detect early lung disease in CF patients. One such method, the Perth-Rotterdam Annotated Grid Morphometric Analysis for CF (PRAGMA-CF), can quantify bronchiectasis, mucus plugging, air trapping and other airway abnormalities in CF patients aged $<6$ years [8]. Lung imaging may be combined with bronchoalveolar lavage (BAL) to survey airway fluid and cells to find associations with structural lung disease. For example, protein-bound glutathione from BAL fluid (BALF) was previously associated with higher risk of developing bronchiectasis [9]. Untargeted analyses have identified dozens of candidate metabolite biomarkers for the detection of CF lung disease [10]. Transition from biomarkers to mechanisms of pathology may lead to new therapeutic strategies to treat early lung disease in CF.

Here, we hypothesised that metabolites in early CF BALF linked to disease progression should correlate with PRAGMA-CF. We applied high-resolution accurate-mass mass spectrometry (MS)-based metabolomics to identify correlations in CF children who underwent prospective BAL and chest CT. We confirmed the identity of the most significant identified metabolite (methionine sulfoxide (MetO)) and quantified MPO, an enzyme from neutrophils capable of generating MetO through production of strong oxidants.

\section{Materials and methods \\ Study design}

The study was performed as part of the Inflammatory Markers in Broncho-Alveolar Lavage Fluid for Lung Disease in Infants with CF (I-BALL) study (ClinicalTrials.gov identifier NCT02907788). It is a translational, exploratory and observational study of infants with CF identified by newborn screening in which BALF, peripheral blood, chest CT and clinical follow-up data are collected prospectively. The Institutional Review Board of the Erasmus Medical Center (Rotterdam, The Netherlands) approved the study (protocol NL49725.078.14) and all parents signed informed consent.

\section{Sample collection}

Bronchoscopy and CT were scheduled prospectively following enrolment and took place within 3 consecutive days on average. Patients fasted overnight prior to the BAL procedure, taking place under general anaesthesia. 24 right middle lobe BAL samples were collected from CF patients aged 12-38 months. Sterile saline ( $1 \mathrm{~mL} \cdot \mathrm{kg}^{-1}$ body weight) was instilled and recovered three times, and research samples pooled from second and third aliquots were placed on ice for $\leqslant 2 \mathrm{~h}$ before BALF was isolated by centrifugation at $330 \mathrm{~g}$ and $4^{\circ} \mathrm{C}$ for $5 \mathrm{~min}$, then stored at $-80^{\circ} \mathrm{C}$. The first $\mathrm{BAL}$ aliquot was used for clinical pathology, including bacterial culture and cell counts. Free-breathing chest CT scans were acquired without anaesthesia using a SOMATOM Force ultra-fast scanner (Siemens Healthcare, Camberley, UK). PRAGMA-CF was used for quantitative scoring of percentages of bronchiectasis (PRAGMA-\%Bx) and total lung disease (PRAGMA-\%Dis) [8]. CT scans were interpreted by a single observer blinded to other study information at the time of scoring. PRAGMA-\%Dis was a composite of PRAGMA-\%Bx, mucus plugging and bronchial wall thickening. Additional demographics and findings are given in table 1. 


\begin{tabular}{|c|c|}
\hline Children $^{\#} \mathrm{n}$ & 24 \\
\hline Age months & $31 \pm 11$ \\
\hline \multicolumn{2}{|l|}{ Sex $n$} \\
\hline Female & 16 \\
\hline Male & 8 \\
\hline F508del homozygous & 50.0 \\
\hline Sweat chloride $\mathrm{mmol} \cdot \mathrm{L}^{-1}$ & $92 \pm 28$ \\
\hline Pancreatic insufficiency & 87.5 \\
\hline Using antibiotics at BAL & 91.2 \\
\hline Past antibiotic courses ${ }^{\natural}$ & $2.9 \pm 1.9$ \\
\hline Second-hand smoke & 29.2 \\
\hline BAL neutrophils $^{+}$ & $27.7 \pm 18.9$ \\
\hline BAL macrophages $^{+}$ & $64.6 \pm 19.7$ \\
\hline BAL lymphocytes $^{+}$ & $7.2 \pm 4.7$ \\
\hline BAL eosinophils $^{+}$ & $0.5 \pm 0.8$ \\
\hline Positive BAL culture $^{+}$ & 39.1 \\
\hline Positive for Staphylococcus aureus ${ }^{+}$ & 26.1 \\
\hline Positive for Pseudomonas aeruginosa ${ }^{+}$ & 4.3 \\
\hline
\end{tabular}

Data are mean \pm SD or $\%$, unless otherwise stated. BAL: bronchoalveolar lavage. ${ }^{\#}$ : all subjects received

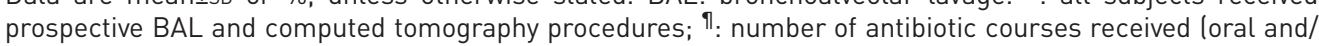
or intravenous, including prophylaxis) 12 months prior to the prospective visit ( $n=18) ;{ }^{+}: n=23$.

\section{Metabolomics}

Metabolites were extracted from BALF by 1:2 mixture with acetonitrile plus internal standards, carried out on ice for $30 \mathrm{~min}$ followed by vortexing and centrifugation at $16000 \mathrm{~g}$ and $4^{\circ} \mathrm{C}$ for $10 \mathrm{~min}$. Supernatant was maintained on an autosampler held at $4^{\circ} \mathrm{C}$ and analysed via a Q Exactive High Field hybrid mass spectrometer (ThermoFisher, Waltham, MA, USA). Intensities of detected mass-to-charge ratio $(\mathrm{m} / \mathrm{z})$ features were extracted using apLCMS version 6.3.3 [11], evaluated for data quality using xMSanalyzer version 2.0.8 [12] and pre-filtered as detailed in the supplementary material. Significant features were annotated using METLIN [13]. Standard Reference Material (SRM) 1950 (National Institute of Standards and Technology (NIST), Gaithersburg, MD, USA) was analysed in parallel to the BALF samples as a quality control and reference standard.

MPO assay

Abundance and activity of MPO were quantified in an assay adapted from CHAPMAN et al. [14]. Briefly, MPO is immunocaptured, and its abundance and activity are measured by ELISA and Amplex Red oxidation, respectively. Assay lower limits of quantification of $1.0 \mathrm{ng} \cdot \mathrm{mL}^{-1}$ (Amplex Red) and $0.13 \mathrm{ng} \cdot \mathrm{mL}^{-1}$ (ELISA) were established from three independent assays.

\section{Additional methods}

Additional methods are given in the supplementary material.

\section{Statistics}

Metabolomics data analysis, including $\log _{2}$ transformation, quantile normalisation, and Pearson's correlation with p-value calculation and false discovery rate-adjusted q-value calculation, was performed using an in-house $\mathrm{R}$ package (https:/github.com/kuppal2/xmsPANDA). We selected $\mathrm{q}<0.25$ for multiple-comparisons adjustment of metabolomics results due to the convolution inherent to electrospray ionisation mass spectra (multiple signals can be observed per compound). Additional analysis was performed using MetaboAnalyst [15] and ggplot2 [16]. MPO assays were calibrated by SoftMax Pro version 7.0.3 (Molecular Devices, San Jose, CA, USA). Prism version 7 (GraphPad, La Jolla, CA, USA) was used to calculate Spearman correlations and the Mann-Whitney U-test, and to generate figures.

\section{Results}

Patient characteristics and CT determination of lung disease

Patient characteristics are shown in table $1.50 \%$ of patients were homozygous for F508del and all but one carried at least one copy of the allele. Three genotyped with an R117H-7T allele and sweat chloride $<60 \mathrm{mmol} \cdot \mathrm{L}^{-1}$ were re-designated as CF screen positive, inconclusive diagnosis (CFSPID; highlighted in red in bar plot and scatter plot figures; range of sweat chloride $24-55 \mathrm{mmol} \cdot \mathrm{L}^{-1}$ ) [17]. Over $90 \%$ of 
patients were on antibiotics at the time of the procedure and less than half of patients were positive for pathogenic organisms by BAL culture.

PRAGMA-CF analysis was conducted on CT scans acquired at 1, 2 or 3 years of age. PRAGMA-CF analysis identified the presence of bronchiectasis in most of the patients (PRAGMA-\%Bx; 0-3.74\%), while the composite score (PRAGMA-\%Dis) ranged from $0.82 \%$ to $7.47 \%$. Clinical variables, such as sex, F508del homozygosity and positive BAL culture, did not significantly influence PRAGMA-\%Dis or we did not consider them because data distributions were unevenly skewed (e.g. pancreatic insufficiency).

\section{Metabolomics}

A list of $11188 \mathrm{~m} / \mathrm{z}$-by-retention time features was initially recovered from BALF samples (supplementary material), and a working feature table of 1798 was produced by applying thresholds for intensity, technical precision and missing values. One sample was determined to have an aberrant number of missing values and removed from the study (supplementary figure S1a and b). Data were then $\log _{2}$-transformed and quantile-normalised prior to testing (supplementary figure S1c). Ultimately, 190 features were significant at $\mathrm{p}<0.05$ and 104 of these passed the $\mathrm{q}<0.25$ multiple-comparisons-adjusted threshold (results in the supplementary material). Significant features were annotated in METLIN [13] followed by isotope analysis and we assigned 30 annotations matching a total of 22 unique metabolites (figure 1 and table 2). In cases of multiple ions belonging to the same metabolite, data for the ion of highest average intensity is shown.

The most significant annotated feature matched to MetO (table 2). Several metabolites corresponded to pathways of arginine metabolism (including arginine, citrulline, ornithine and diacetylspermine) or to glycerophospholipids and lysolipids. Others included lithocholic acid, trigonelline and several other amino acids. Most were positively associated with PRAGMA-\%Dis, but three (phosphatidylcholine 42:6, phosphatidylethanolamine acyl/ether 32:0 and lithocholic acid) were inversely associated. 12 unidentified features ranging from $\mathrm{m} / \mathrm{z} 675$ to 861 and co-eluting at $330 \mathrm{~s}$ showed strong positive correlations with PRAGMA-\%Dis (supplementary material; $r>0.6, \mathrm{p} \leqslant 0.001$ for all), but these produced no reasonable database matches and were not further assessed.

\section{Identification and reference calibration of MetO}

We sought to confirm the accurate-mass annotation of MetO (figure 1 and table 2; representative trace in supplementary figure S2a). We synthesised isotopically enriched ${ }^{13} \mathrm{C}_{5},{ }^{15} \mathrm{~N}$-L-MetO and spiked this into pooled CF BALF and SRM 1950 to identify MetO by stable isotope co-elution and MS/MS. Naturally occurring and isotopically enriched MetO gave rise to the same five fragments with appropriate mass shifts in the isotopically enriched reagent (supplementary figures S2b and c), providing confirmation of the two-dimensional structure of MetO in both BALF and SRM 1950 (supplementary figure S2d).

We calibrated MetO and methionine in SRM 1950 to enable reference calibration of the BALF samples and calculate the percentage of MetO relative to the sum of MetO and methionine (\%OxMet). SRM 1950 was calibrated at $20.68 \mu \mathrm{M}$ methionine (93\% of the NIST-certified reference value, $22.3 \mu \mathrm{M}$ ) and $1.22 \mu \mathrm{M}$ MetO, corresponding to 5.6\% OxMet. MetO was then reference calibrated in BALF and ranged from 24 to $1031 \mathrm{nM}$ (mean \pm SD $174 \pm 240 \mathrm{nM}$ ), while \%OxMet ranged from $3.8 \%$ to $62.7 \%$ (mean \pm SD $18.8 \pm 14.8 \%$ ). After calibration, MetO remained significantly correlated with PRAGMA-\%Dis using nonparametric Spearman correlation (figure 2a).

\section{Quantification of MPO in CF BALF}

MetO is produced by the reaction of methionine with MPO-derived oxidants hypochlorous $(\mathrm{HOCl})$ and hypobromous acid (HOBr) [18]. MPO was detected in BALF and exhibited the same immunoreactivity as in blood neutrophils (supplementary figure S3). We determined the catalytic activity and protein abundance of MPO in CF BALF to evaluate its potential relationships with PRAGMA-\%Dis and MetO. MPO ranged from 0.0087 to $4.5 \mu \mathrm{g} \cdot \mathrm{mL}^{-1}$ in CF BALF (mean \pm sD $0.90 \pm 1.17 \mu \mathrm{g} \cdot \mathrm{mL}^{-1}$ ), except for one CFSPID sample in which none was detected. MPO distribution was nonnormal and was similar when normalised to protein (supplementary figure S4a). BALF MPO was active in an Amplex Red-based peroxidase assay and none of the samples exhibited low activity percentage relative to ELISA results (supplementary figure S4b and c). BALF MPO correlated strongly with PRAGMA-\%Dis (figure 2b), as well as MetO (figure 2c).

\section{Relationships of MetO and MPO with bronchiectasis and neutrophils}

We sought to understand the specific contributions of bronchiectasis (PRAGMA-\%Bx) and the percentage of airway neutrophils to MetO, \%OxMet and MPO. We performed pairwise Spearman correlations for these variables (table 3). For each of the variables, including airway neutrophils, PRAGMA-\%Bx resulted in 

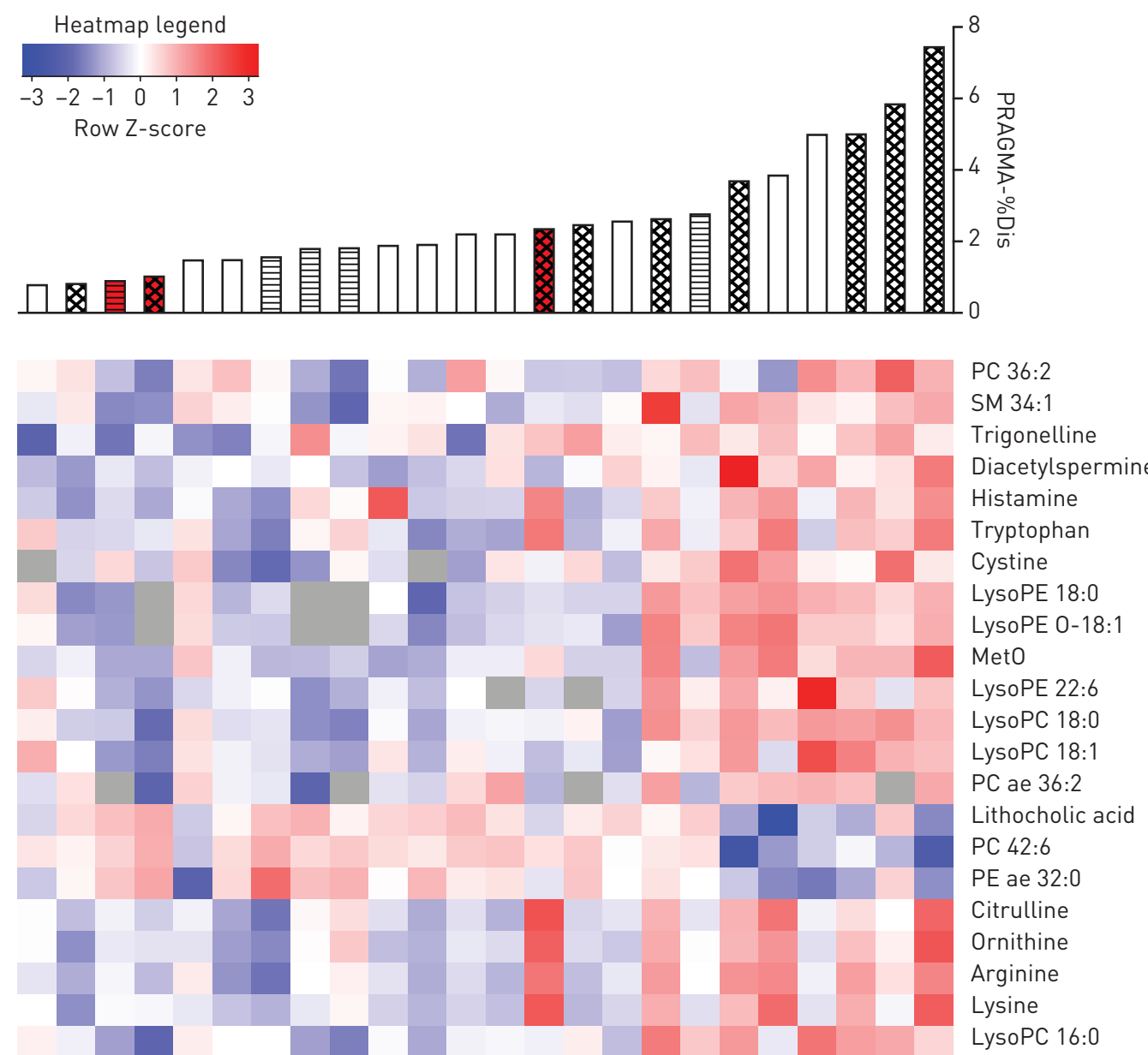

FIGURE 1 Accurate-mass metabolite annotations of mass-to-charge ratio $(\mathrm{m} / \mathrm{z})$ features significantly associated with Perth-Rotterdam Annotated Grid Morphometric Analysis for Cystic Fibrosis (PRAGMA-CF) percentage of total lung disease (PRAGMA-\%Dis). PC: phosphatidylcholine; SM: sphingomyelin; PE: phosphatidylethanolamine; Met0: methionine sulfoxide; ae: acyl/ether; BAL: bronchoalveolar lavage. Intensities of $\mathrm{m} / \mathrm{z}$-by-retention time features measured from CF BAL fluid by high-resolution accurate-mass mass spectrometry were $\log _{2}$-transformed, quantile-normalised and correlated with PRAGMA-\%Dis using a linear model. Of 104 significant $(p<0.05, q<0.25)$ features, 30 were annotated to accurate-mass matches using METLIN [13] followed by isotope analysis. These corresponded to 22 unique metabolites after deconvolution of co-eluting compounds (see also table 2 and the complete dataset given in the supplementary material). The $\mathrm{m} / \mathrm{z}$ feature with highest average intensity for each of the 22 metabolites was plotted on a heatmap, autoscaled to Z-scores and coded blue (most negative) to red (most positive). Missing values are represented as grey. Columns were sorted from left-to-right by ascending PRAGMA-\%Dis and rows were hierarchically clustered by metabolite intensities. Accurate-mass metabolite matches are indicated to the right of the heatmap. PRAGMA-\%Dis values corresponding to each patient are shown by a bar plot above the heatmap. Bar colour corresponds to disease state (white: CF; red: CF screen positive, inconclusive diagnosis). Bar fill corresponds to BAL microbe culture (no fill: no history of positive pathogen culture; diagonal fill: negative for pathogens at time of BAL, but a prior BAL was positive; hatched fill: positive pathogen culture at time of BAL).

a stronger correlation than PRAGMA-\%Dis. The strongest observed correlation between two independent variables was that of \%OxMet and MPO.

\section{Robustness of key correlations}

To ensure robustness of key correlations, we analysed a subset of patients by excluding three CFSPID-designated samples and five others for whom clinical complications prevented the completion of BAL and CT procedures within 28 consecutive days. After removing the total eight samples, MetO remained significantly correlated with PRAGMA-\%Bx and MPO, but the significant association with PRAGMA-\%Dis was lost (supplementary figure S5). As the strength of the subset correlation $(\rho=0.4353)$ was comparable to the original $(\rho=0.5817)$, decreased power probably explains the lack of significance. MPO remained significantly correlated with PRAGMA-\%Dis and PRAGMA-\%Bx (supplementary figure S4d and e). 
TABLE 2 Metabolites correlated with Perth-Rotterdam Annotated Grid Morphometric Analysis for Cystic Fibrosis (PRAGMA-CF) percentage of total lung disease in untargeted analysis

\begin{tabular}{lccccccc}
$\mathbf{m} / \mathbf{z}$ & Time $\mathbf{s}$ & Metabolite & Adduct & Error ppm & $\mathbf{r}$ & $\mathbf{p}$-value & $\mathbf{q}$-value \\
\hline $\mathbf{1 6 6 . 0 5 3 3}$ & 281 & MetO & {$[\mathrm{M}+\mathrm{H}]$} & 0 & 0.648 & $5.59 \times 10^{-5}$ & 0.0273 \\
$\mathbf{8 6 2 . 6 3 3 9}$ & 137 & PC 42:6 & {$[\mathrm{M}+\mathrm{H}]$} & 2 & -0.357 & $9.89 \times 10^{-5}$ & 0.0273 \\
$\mathbf{5 2 4 . 3 7 1 1}$ & 270 & LysoPC 18:0 & {$[\mathrm{M}+\mathrm{H}]$} & 0 & 0.669 & $3.57 \times 10^{-4}$ & 0.0407 \\
$\mathbf{2 8 7 . 2 4 4 2}$ & 320 & Diacetylspermine & {$[\mathrm{M}+\mathrm{H}]$} & 0 & 0.318 & $6.36 \times 10^{-4}$ & 0.0425 \\
$\mathbf{5 2 2 . 3 5 5 6}$ & 270 & LysoPC 18:1 & {$[\mathrm{M}+\mathrm{H}]$} & 0 & 0.599 & $2.69 \times 10^{-3}$ & 0.1272 \\
$\mathbf{1 7 5 . 1 1 9 0}$ & 305 & Arginine & {$[\mathrm{M}+\mathrm{H}]$} & 0 & 0.254 & $2.78 \times 10^{-3}$ & 0.1272 \\
$\mathbf{4 9 6 . 3 3 9 7}$ & 274 & LysoPC 16:0 & {$[\mathrm{M}+\mathrm{H}]$} & 0 & 0.598 & $2.86 \times 10^{-3}$ & 0.1272 \\
$\mathbf{1 3 3 . 0 9 7 2}$ & 308 & Ornithine & {$[\mathrm{M}+\mathrm{H}]$} & 0 & 0.304 & $2.98 \times 10^{-3}$ & 0.1277 \\
$\mathbf{4 8 2 . 3 2 4 0}$ & 196 & LysoPE 18:0 & {$[\mathrm{M}+\mathrm{H}]$} & 0 & 0.579 & $3.70 \times 10^{-3}$ & 0.1428 \\
$\mathbf{4 6 6 . 3 2 8 8}$ & 192 & LysoPE 0-18:1 & {$[\mathrm{M}+\mathrm{H}]$} & 0 & 0.640 & $4.80 \times 10^{-3}$ & 0.1586 \\
$\mathbf{1 4 7 . 1 1 2 8}$ & 311 & Lysine & {$[\mathrm{M}+\mathrm{H}]$} & 0 & 0.197 & $6.72 \times 10^{-3}$ & 0.1920 \\
$\mathbf{1 7 6 . 1 0 3 0}$ & 257 & Citrulline & {$[\mathrm{M}+\mathrm{H}]$} & 0 & 0.307 & $8.68 \times 10^{-3}$ & 0.2061 \\
$\mathbf{1 1 2 . 0 8 6 9}$ & 303 & Histamine & {$[\mathrm{M}+\mathrm{H}]$} & 0 & 0.284 & $9.54 \times 10^{-3}$ & 0.2152 \\
$\mathbf{8 0 8 . 5 8 1 7}$ & 215 & PC 36:2 & {$[\mathrm{M}+\mathrm{Na}]$} & 1 & 0.161 & 0.0127 & 0.2314 \\
$\mathbf{4 1 5 . 2 6 0 7}$ & 222 & Lithocholic acid & {$[\mathrm{M}+\mathrm{K}]$} & 0 & -0.193 & 0.0141 & 0.2314 \\
$\mathbf{5 2 6 . 2 9 1 3}$ & 192 & LysoPE 22:6 & {$[\mathrm{M}+\mathrm{H}]$} & 2 & 0.310 & 0.0159 & 0.2431 \\
$\mathbf{6 6 0 . 5 3 3 2}$ & 157 & PE ae 32:0 & {$[\mathrm{M}+\mathrm{H}-\mathrm{H}+\mathrm{O}]$} & 0 & -0.049 & 0.0183 & 0.2456 \\
$\mathbf{7 7 2 . 6 2 3 2}$ & 218 & PC ae 36:2 & {$[\mathrm{M}+\mathrm{H}]$} & 2 & 0.678 & 0.0186 & 0.2456 \\
$\mathbf{2 0 5 . 0 9 7 1}$ & 182 & Tryptophan & {$[\mathrm{M}+\mathrm{H}]$} & 0 & 0.309 & 0.0198 & 0.2467 \\
$\mathbf{7 0 3 . 5 7 2 9}$ & 247 & SM 34:1 & {$[\mathrm{M}+\mathrm{H}]$} & 2 & 0.150 & 0.0205 & 0.2467 \\
$\mathbf{2 4 1 . 0 3 1 1}$ & 306 & Cystine & {$[\mathrm{M}+\mathrm{H}]$} & 0 & 0.420 & 0.0207 & 0.2467 \\
$\mathbf{1 3 8 . 0 5 5 0}$ & 267 & Trigonelline & {$[\mathrm{M}+\mathrm{H}]$} & 0 & 0.153 & 0.0221 & 0.2467 \\
& & & & & &
\end{tabular}

$\mathrm{m} / \mathrm{z}$ : mass-to-charge ratio; MetO: methionine sulfoxide; PC: phosphatidylcholine; PE: phosphatidylethanolamine; ae: acyl/ether; SM: sphingomyelin. Metabolites $p<0.05, q<0.25$ are shown following deconvolution to remove redundant features that correspond to the same chemical. In such cases, the $\mathrm{m} / \mathrm{z}$ feature with the highest mean intensity was selected.

\section{Global correlations}

To compare the global correlations of MetO, MPO and PRAGMA-CF to clinical variables, we prepared a Spearman correlation matrix (figure 3). We considered BAL immune cell percentages, BALF total protein, patient age, sweat chloride and the number of antibiotic courses. The three strongest correlations with PRAGMA-\%Dis were MPO, MetO and BAL percentage of macrophages $(\rho=-0.5824, p=0.0036)$; the three strongest correlations with PRAGMA-\%Bx were MPO, MetO and BAL percentage of neutrophils. BAL percentage of neutrophils and BAL percentage of macrophages exhibited a strong inverse relationship $(\rho=$
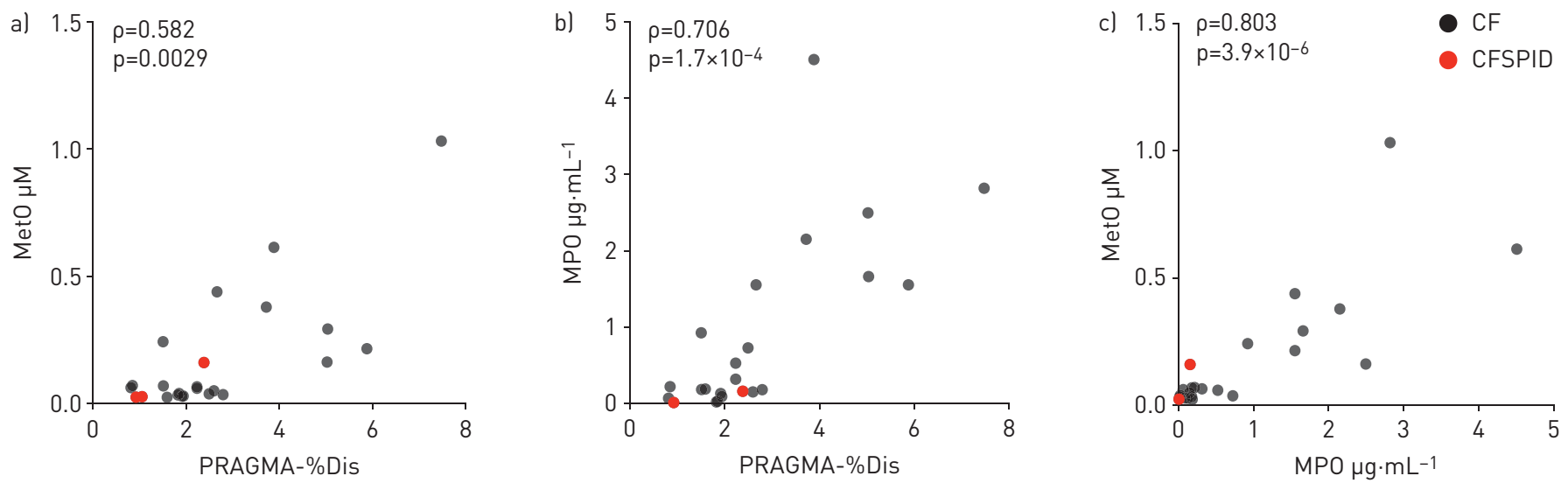

FIGURE 2 Correlations of structural lung disease, methionine oxidation and myeloperoxidase (MPO) in early cystic fibrosis (CF): Spearman correlations were analysed for pairwise combinations of $a$, b) Perth-Rotterdam Annotated Grid Morphometric Analysis for CF (PRAGMA-CF) percentage of total lung disease (PRAGMA-\%Dis), a, c) methionine sulfoxide (MetO) (measured by high-resolution accurate-mass mass spectrometry with reference calibration) and b, c) MPO (measured by ELISA). CFSPID: CF screen positive, inconclusive diagnosis. The correlation results ( $\rho$ and $p$-value) are shown for each scatter plot. CFSPID patients, identified by newborn screening with later inconclusive diagnosis of disease, are indicated. 
TABLE 3 Pairwise correlations of variables associated with lung disease

\begin{tabular}{|c|c|c|c|c|c|}
\hline & PRAGMA-\%Bx & PMNs \% & Met0 nM & $\% 0 x M e t$ & MPO $\mu \mathrm{g} \cdot \mathrm{mL}^{-1}$ \\
\hline PRAGMA-\%Dis & $\begin{array}{c}n=24 \\
\rho=0.726 \\
p=5.8 \times 10^{-5}\end{array}$ & $\begin{array}{c}n=23 \\
\rho=0.502 \\
p=0.0146\end{array}$ & $\begin{array}{c}n=24 \\
\rho=0.582 \\
p=0.0029\end{array}$ & $\begin{array}{c}n=24 \\
\rho=0.437 \\
p=0.0286\end{array}$ & $\begin{array}{c}n=23 \\
\rho=0.706 \\
p=1.7 \times 10^{-4}\end{array}$ \\
\hline PRAGMA-\%Bx & & $\begin{array}{c}n=23 \\
\rho=0.6742 \\
p=4.2 \times 10^{-4}\end{array}$ & $\begin{array}{c}n=24 \\
\rho=0.698 \\
p=1.5 \times 10^{-4}\end{array}$ & $\begin{array}{c}n=24 \\
\rho=0.665 \\
p=3.9 \times 10^{-4}\end{array}$ & $\begin{array}{c}n=23 \\
\rho=0.752 \\
p=3.5 \times 10^{-5}\end{array}$ \\
\hline PMNs \% & & & $\begin{array}{c}n=23 \\
\rho=0.569 \\
p=0.0046\end{array}$ & $\begin{array}{c}n=23 \\
\rho=0.594 \\
p=0.0028\end{array}$ & $\begin{array}{c}n=22 \\
\rho=0.656 \\
p=0.0092\end{array}$ \\
\hline Met0 nM & & & & $\begin{array}{c}n=24 \\
\rho=0.860 \\
p=7.2 \times 10^{-8}\end{array}$ & $\begin{array}{c}n=23 \\
\rho=0.803 \\
p=3.9 \times 10^{-6}\end{array}$ \\
\hline$\% 0 x M e t$ & & & & & $\begin{array}{c}n=23 \\
\rho=0.837 \\
p=6.4 \times 10^{-7}\end{array}$ \\
\hline
\end{tabular}

Pairwise Spearman correlation results are shown for the listed variables. PRAGMA-CF: Perth-Rotterdam Annotated Grid Morphometric Analysis for Cystic Fibrosis; PRAGMA-\%Dis: PRAGMA-CF percentage of total lung disease; PRAGMA-\%Bx: PRAGMA-CF percentage of bronchiectasis; PMN: polymorphonuclear neutrophil; MetO: methionine sulfoxide; \%OxMet: percentage of MetO relative to the sum of MetO and methionine; MPO: myeloperoxidase. Note that the variable pairs of PRAGMA-\%Dis and PRAGMA-\%Bx and of MetO and \%OxMet are not independent (PRAGMA-\%Bx contributes directly to PRAGMA-\%Dis; MetO contributes directly to \%OxMet).

-0.9683, $\left.\mathrm{p}=3.9 \times 10^{-14}\right)$. Age and BALF protein also had positive correlations with PRAGMA-\%Dis and PRAGMA-\%Bx. BAL percentage of eosinophils, BAL percentage of lymphocytes, sweat chloride and the number of antibiotic courses in the past 12 months were not correlated with other variables.

a)

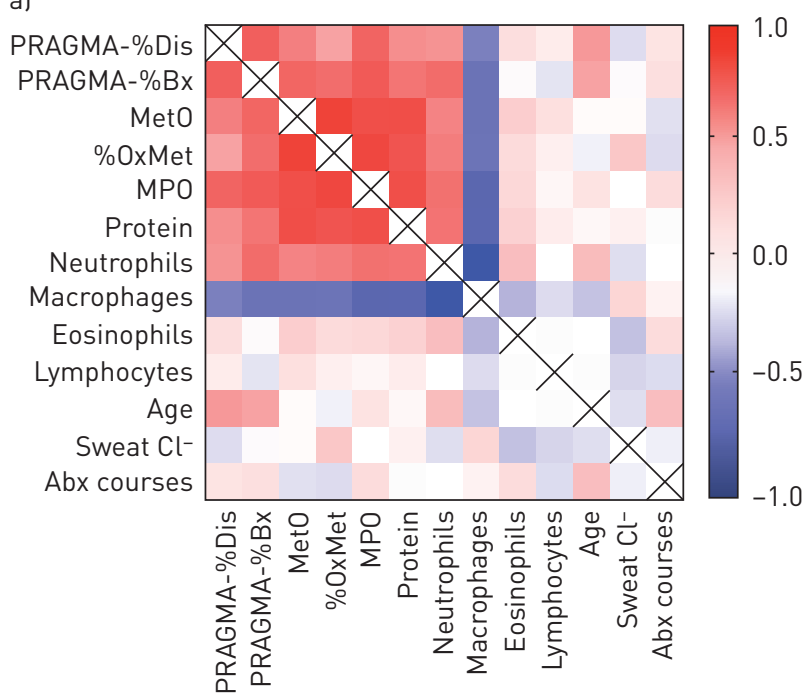

b)

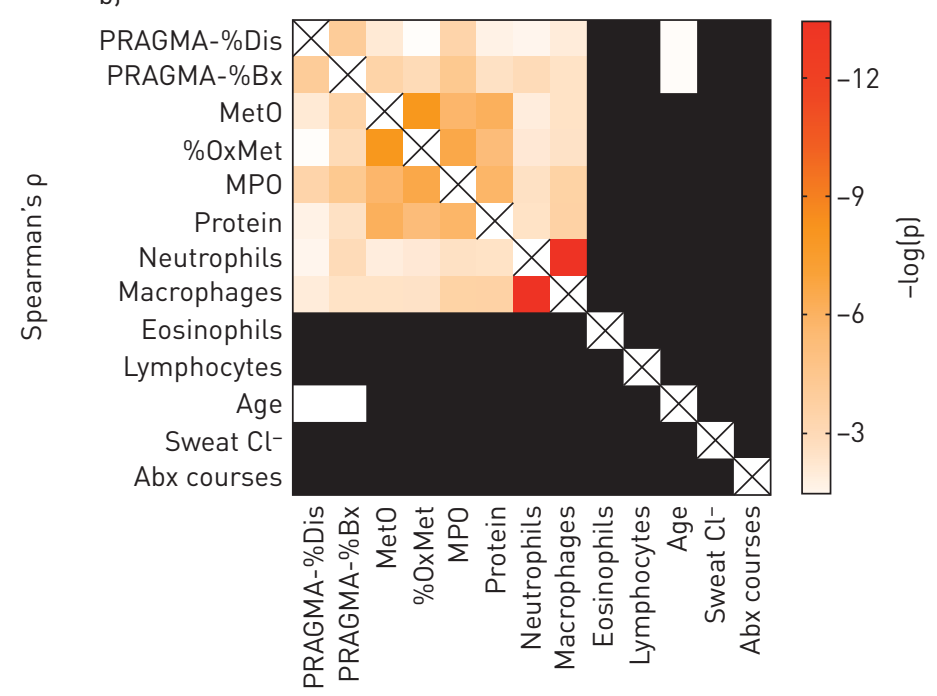

FIGURE 3 Methionine oxidation, airway myeloperoxidase (MPO) and neutrophils are strong correlates of bronchiectasis in early cystic fibrosis (CF). PRAGMA-CF: Perth-Rotterdam Annotated Grid Morphometric Analysis for CF; PRAGMA-\%Dis: PRAGMA-CF percentage of total lung disease; PRAGMA-\%Bx: PRAGMA-CF percentage of bronchiectasis; Met0: methionine sulfoxide; \%OxMet: percentage of Met0 relative to the sum of Met0 and methionine; $\mathrm{Cl}^{-}$: chloride; Abx: antibiotic. We tested the Spearman correlations of PRAGMA-\%Dis and PRAGMA-\%Bx with BALF measures (Met0, \%OxMet, MPO and total protein), bronchoalveolar lavage cell populations (percentages of neutrophils, macrophages, lymphocytes and eosinophils), patient age (months), patient sweat $\mathrm{Cl}^{-}(\mathrm{mM})$ and the total number of Abx courses received by patients in the prior 12 months. a) Blue-to-red heatmap of Spearman's $\rho$ for the indicated variables (red: positive correlation; blue: negative correlation). The intensity of the colour indicates the strength of the relationship. b) Matching single-gradient white-to-red heatmap of p-values (expressed as -loglp)). Intensity of the red colour indicates greater statistical significance. Black cells indicate results of $p>0.05$ for the relevant pairwise correlation. Cells denoted by " $x$ " indicate where rows and columns for the same variable meet. 


\section{Discussion}

Taken together, our results are consistent with the early emergence of neutrophilic influx, granule exocytosis and oxidising activity of secreted MPO early in CF pathology, particularly bronchiectasis (scheme in figure 4). The contribution of neutrophilic inflammation to CF lung disease is well recognised, yet exact mechanisms of pathogenesis are not fully understood. Interventions directly targeting inflammation in CF are currently limited to high-dose ibuprofen and no US Food and Drug Administration-approved therapies specifically target airway neutrophils [19]. Such an intervention might be most effective and have the longest lasting benefits if administered in the earliest stages of CF [20]. It is not yet known how CFTR modulators will influence inflammation in the lungs and these drugs are not yet available for young children.

The present study demonstrates the potential importance of methionine oxidation by MPO in early CF lung disease. Airway MetO and \%OxMet strongly correlated with MPO, indicating that MPO is responsible for the MetO detected. Biomarker studies have indicated that $\mathrm{MPO}$ does generate $\mathrm{HOCl}$ and $\mathrm{HOBr}$ in $\mathrm{CF}$ airways [21]. Distinct oxidants with diverse reactivity profiles are generated in the airways that may serve to help or harm the host, with $\mathrm{HOCl}$ and $\mathrm{HOBr}$ associated with lasting molecular damage [22]. These oxidants have exceptional reduction potentials and rapidly oxidise methionine [18, 23], while weaker oxidants and those that are kinetically restrained, such as hypothiocyanous acid and hydrogen peroxide $\left(\mathrm{H}_{2} \mathrm{O}_{2}\right)$, respectively, react much more slowly with methionine, so that their contributions to its oxidation in vivo may effectively be nil $[24,25]$. The strong correlation observed for MPO and \%OxMet in this cohort supports the notion that $\mathrm{MPO}$ generates significant $\mathrm{HOCl}$ and $\mathrm{HOBr}$ in $\mathrm{CF}$ airways at a young age. Importantly, MPO may do this regardless of the source of $\mathrm{H}_{2} \mathrm{O}_{2}$, which is generated in vivo from multiple physiological processes [26]. Although phagocyte NADPH oxidase 2 is a likely contributor to neutrophil-derived $\mathrm{H}_{2} \mathrm{O}_{2}$ (via superoxide), other sources of $\mathrm{H}_{2} \mathrm{O}_{2}$ should not be ruled out.

While the influence of MPO is well recognised in CF airway pathophysiology $[9,21]$, this study is, to the best of our knowledge, the first time that $\mathrm{MetO}$ and \%OxMet have been identified as correlates of bronchiectasis and airway neutrophils in young children with CF, which we discovered using an unbiased metabolomics approach. Of note, untargeted mass spectral data may incur multiple-comparisons penalties that are ultimately too conservative, as multiple interdependent spectra can arise from individual chemicals in the samples [27], resulting in a higher likelihood of type II error [28]. Recognising this, we used a moderate multiple-comparisons threshold of $\mathrm{q}<0.25$ for untargeted mass spectral data. However, we note that multiple metabolites, including MetO, would have surpassed more stringent thresholds. Although our data suggest that methionine is one of the most sensitive airway metabolites to the advancement of

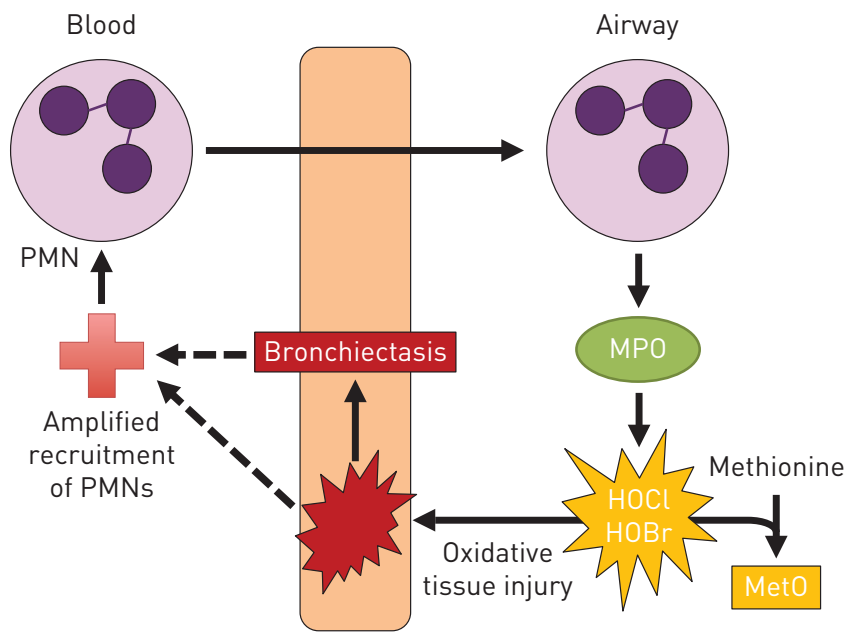

FIGURE 4 Roles of myeloperoxidase (MPO) and methionine sulfoxide (MetO) in the development of early cystic fibrosis (CF) bronchiectasis. PMN: polymorphonuclear neutrophil; $\mathrm{HOCl}$ : hypochlorous acid; $\mathrm{HOBr}$ : hypobromous acid. PMN recruitment to airways may occur in early CF without an obvious pathogenic stimulus. In the current study, MetO in early CF bronchoalveolar lavage fluid was correlated strongly with computed tomography scores of bronchiectasis. Following influx of neutrophils that secrete MPO into the lumen, the oxidising activity of MPO may generate MetO from methionine. MPO generates $\mathrm{HOCl}$ and $\mathrm{HOBr}$, which cause rapid, frequently irreversible damage to an array of biomolecules, including tissue macromolecules. The oxidising activity of MPO provides a common link between MetO and tissue injury which may lead to bronchiectasis. These processes may be insufficiently resolved in early CF and contribute to a feed-forward mechanism underlying sustained PMN recruitment. Whether MetO may directly participate in the promotion of CF airway disease is unclear at this time. 
neutrophilia and bronchiectasis in early CF, additional studies are needed to validate the importance of methionine oxidation in early CF. In addition to these findings, our study confirms the relationship of \%OxMet and MPO in CF BALF previously identified by DicKerHof et al. [9].

To maximise clinical utility, airway $\mathrm{MetO}$ and \%OxMet measurements should be extended in future studies to include less invasive samples, such as exhaled breath condensate (EBC), sputum and tracheal aspirates. While we anticipate that sputum and tracheal aspirates will be sufficiently concentrated to perform robust metabolomics research, methodological innovations to improve sensitivity for dilute EBC samples are a necessity. Fortunately, MS has already been demonstrated to be amenable to CF EBC metabolite detection [29]. Standardised collection in accordance with best clinical practice is also crucial, particularly in early-stage paediatric studies where reproducible sample acquisition can be challenging.

Correlations of MetO and \%OxMet with bronchiectasis are consistent with previously hypothesised redox dysregulation and oxidative stress in CF. Much emphasis has been placed on the decreased abundance and accelerated oxidation of glutathione in $\mathrm{CF}$ airways [9, 30, 31]. By contrast, we found that cystine (the homodimeric disulfide of cysteine) was positively correlated with PRAGMA-\%Dis. As the samples were not treated to preserve thiols, the result may be indicative of the total cysteine and cystine pool rather than the oxidised form alone. This indicates that glutathione and cysteine likely support divergent redox signalling pathways in airways, much like other physiological compartments [32]. We did not detect the irreversibly oxidised glutathione sulfonamide in the current study, which may be due to methodological differences and/or reflect its low abundance, possibly limited by its stringent reaction $(3 \mathrm{~mol} \mathrm{HOCl}$ or $\mathrm{HOBr}$ react with $1 \mathrm{~mol}$ glutathione) [33].

The association of MetO and early CF bronchiectasis could result from multiple pathophysiological processes. Excessive and sustained neutrophil transmigration is associated with progressive airway damage [34] and is a necessary precursor to the accumulation of luminal MPO required for efficient methionine oxidation by $\mathrm{HOCl} / \mathrm{HOBr}$. However, neutrophils secrete several other potentially damaging factors and MetO could represent multiple pathways of damage by proxy. For example, MPO release is generally concomitant with that of neutrophil elastase [4]. However, additional experiments are needed to establish the relationship of these enzymes in early CF. In particular, future studies will assess whether MPO-dependent MetO production may be readily measurable in infants with CF while elastase activity may not, due to the airway antiprotease shield that counterbalances elastase effectively early on in the disease process [3].

In contrast to MetO, which associated more strongly with PRAGMA-\%Bx than PRAGMA-\%Dis, methionine was the opposite: it was associated more strongly with PRAGMA-\%Dis than with PRAGMA-\%Bx (however, neither association was as strong as those of MetO; data not shown). This reversal may reflect the association of methionine with different disease processes not directly related to MPO activity, such as bulk movement of metabolites into the airway lumen with neutrophils or the methionine salvage pathway. The methionine salvage pathway, in which methionine and adenine are recovered from byproducts of polyamine biosynthesis, has been implicated in worse lung function in $\mathrm{CF}$ by genome-wide association and transcriptomic studies indicating potential roles for AMD1 (adenosylmethionine decarboxylase 1), MTAP (methylthioadenosine phosphorylase) and APIP (APAF1 interacting protein) [35-37]. Airway methionine oxidation might promote increased expression of this pathway and such potential for pathological cross-talk should be considered in future studies. Additional studies are also needed to determine the extent to which the steady-state redox potential of methionine is actively maintained in airways, including individual fates of the MetO stereoisomers, i.e. methionine- $S$-sulfoxide and methionine- $R$-sulfoxide. These are reduced back to methionine by distinct enzymes [38], although mammals do not encode an efficient free methionine- $R$-sulfoxide reductase [39].

Inflammation in CF may be present from an extremely early age, provoked by inherent epithelial defects [7]. A recent study in children $<18$ years of age showed a decreased rate of annual lung function decline and increased long-term survival in high-dose ibuprofen-exposed patients [40]. The effects of high-dose ibuprofen may include inhibition of neutrophil oxidative burst [41], which would in turn limit production of $\mathrm{HOCl}$ and $\mathrm{HOBr}$ from MPO. Many compounds have been investigated as candidate MPO inhibitors, including 2-thioxanthines and acetaminophen $[42,43]$. Such drugs may find utility in limiting early CF lung disease, but this requires further testing. Other interventions directly targeting key reactive species such as $\mathrm{HOCl}$ may also have potential to limit airway disease caused by hypohalous acids [44-47]. Strategies to limit oxidative imbalance in CF should take the species of oxidants generated and their respective roles into account, as oxidants serve diverse and critical roles in host defence and redox signalling processes [22, 26].

We included three patients with R117H-7T mutations, designated CFSPID after initial enrolment via newborn screening, in the study. Interestingly one of the patients showed significant inflammation, heightened MetO and increased PRAGMA-CF scores on chest CT, despite having a mutation which is considered as not causing CF. Therefore, PRAGMA-CF and BAL studies that are useful in identifying lung 
disease processes in CF appear to have some applicability to CFSPID as well. Future studies may identify environmental and genetic factors that promote neutrophilic lung disease in otherwise healthy CFSPID and/or in well-controlled CF patients.

In conclusion, we present data that demonstrate the importance of methionine oxidation via MPO in early CF airway disease. MetO, \%OxMet and MPO strongly correlated with PRAGMA-\%Dis and PRAGMA-\%Bx, sensitive measures of early lung disease and bronchiectasis. Our initial discovery was made through an unbiased metabolomics method and confirmed using targeted analyses. MetO and MPO should be further studied in pathological mechanisms underlying early onset CF airway disease, which may lead to novel biomarkers and targets for therapy.

Acknowledgements: We thank the patients and their families for consenting to participate in this study. We acknowledge research coordinators E. Nieuwhof, E. van der Wiel and B. Manai (Erasmus MC-Sophia, Rotterdam, The Netherlands) for their support in recruiting patients. We thank M.W.H. Pijnenburg, J.C. de Jongste, L. Duijts, S. Kloosterman and I.M. de Kleer (Erasmus MC-Sophia, Rotterdam, The Netherlands) for performing bronchoscopies. We thank M. Kemner (Erasmus MC-Sophia, Rotterdam, The Netherlands) for her guidance with PRAGMA-CF scoring. We thank Nina Dickerhof (University of Otago, Christchurch, New Zealand) for insightful technical discussions about the MPO assay method.

Author contributions: Experimental conception and design: R. Tirouvanziam, H.M. Janssens, B.J. Scholte and J.D. Chandler. Performed experiments: J.D. Chandler, H. Horati, C. Margaroli, M.B. Kilgore and M. Veltman. Interpreted results and provided critical support: J.D. Chandler, H. Horati, C. Margaroli, M.B. Kilgore, H.K. Liu, B.J. Scholte, H.A.W.M. Tiddens, A.J. Taurone, L. Peng, L. Guglani, K. Uppal, Y-M. Go, H.M. Janssens, D.P. Jones and R. Tirouvanziam. Prepared manuscript: J.D. Chandler. Reviewed or edited and approved manuscript: all authors.

Conflict of interest: J.D. Chandler reports grants from NIH and Cystic Fibrosis Foundation, outside the submitted work. C. Margaroli reports grants from NIH (R01HL126603) and CF@LANTA RDP Fellowship, during the conduct of the study. H. Horati reports grants from NIH, outside the submitted work. M.B. Kilgore has nothing to disclose. M. Veltman has nothing to disclose. H.K. Liu has nothing to disclose. A.J. Taurone has nothing to disclose. L. Peng reports grants from NIH, outside the submitted work. L. Guglani has nothing to disclose. K. Uppal has nothing to disclose. Y-M. Go has nothing to disclose. H.A.W.M. Tiddens participated in an industry symposium on treatment in cystic fibrosis for Roche, has performed lectures and advisory board work for Novartis and Gilead, has received grants from CFF and Chiesi, and has received grants from and performed advisory board work for Vertex, outside the submitted work; in addition, H.A.W.M. Tiddens has a patent Vectura licensed, and a patent PRAGMA-CF scoring system issued, and is head of the Erasmus MC-Sophia Children's Hospital core laboratory Lung Analysis. B.J. Scholte reports grants from ERARE (INSTINCT), ZonMw (43000035) and NIH (1R01HL126603-01), during the conduct of the study; a previous study describing S1P metabolism in CF mutant mice was partially funded by a research grant and compound (S1P lyase inhibitor) from Lexicon Pharmaceuticals, Inc., The Woodlands, TX, USA. The MTA granted Erasmus MC unconditional publication rights. R. Tirouvanziam reports grants from NIH (R01HL126603 and R01HL126603-02S1), during the conduct of the study; and personal fees (as scientific advisory board member) from Celtaxsys, Inc., outside the submitted work. D.P. Jones has nothing to disclose. H.M. Janssens reports grants from NIH and Dutch CF foundation, during the conduct of the study; and has received speaker fees (paid to Sophia Research office) from Vertex, and grants from Sophia Foundation for research, outside the submitted work.

Support statement: Supported by NHLBI F32 HL132493 (J.D. Chandler); Emory Children's CF and Airways Disease Research Center Startup Fund (J.D. Chandler and M.B. Kilgore); CFF P30 MCCART15R0 (C. Margaroli); NHLBI R01 HL126603 and Common Fund for Metabolomics Supplement HL126603-02S1 (R. Tirouvanziam, A.J. Taurone, L. Peng, H. Horati, H.M. Janssens, B.J. Scholte and M. Veltman); Dutch Cystic Fibrosis Foundation (NCFS) grant HIT-CF 1 and 2 (H. Horati, M. Veltman, B.J. Scholte, H.A.W.M. Tiddens and H.M. Janssens); ERARE INSTINCT (M. Veltman and B.J. Scholte); and P30 ES019776, S10 OD018006 and R01 ES023485 (H.K. Liu, K. Uppal, Y-M. Go and D.P. Jones). Funding information for this article has been deposited with the Crossref Funder Registry.

\section{References}

$1 \quad$ Elborn JS. Cystic fibrosis. Lancet 2016; 388: 2519-2531.

2 Stoltz DA, Meyerholz DK, Welsh MJ. Origins of cystic fibrosis lung disease. N Engl J Med 2015; 372: 351-362.

3 Sly PD, Gangell CL, Chen L, et al. Risk factors for bronchiectasis in children with cystic fibrosis. N Engl J Med 2013; 368: 1963-1970.

4 Margaroli C, Tirouvanziam R. Neutrophil plasticity enables the development of pathological microenvironments: implications for cystic fibrosis airway disease. Mol Cell Pediatr 2016; 3: 38.

5 Hartl D, Gaggar A, Bruscia E, et al. Innate immunity in cystic fibrosis lung disease. J Cyst Fibros 2012; 11: 363-382.

6 Forrest OA, Ingersoll SA, Preininger MK, et al. Frontline science: pathological conditioning of human neutrophils recruited to the airway milieu in cystic fibrosis. J Leukoc Biol 2018; 16: 45.

7 Montgomery ST, Mall MA, Kicic A, et al. Hypoxia and sterile inflammation in cystic fibrosis airways: mechanisms and potential therapies. Eur Respir J 2017; 49: 1600903.

8 Rosenow T, Oudraad MCJ, Murray CP, et al. A quantitative structural lung disease computed tomography outcome in young children with cystic fibrosis. Am J Respir Crit Care Med 2015; 191: 1158-1165.

9 Dickerhof N, Pearson JF, Hoskin TS, et al. Oxidative stress in early cystic fibrosis lung disease is exacerbated by airway glutathione deficiency. Free Radic Biol Med 2017; 113: 236-243.

10 Esther CR, Turkovic L, Rosenow T, et al. Metabolomic biomarkers predictive of early structural lung disease in cystic fibrosis. Eur Respir J 2016; 48: 1612-1621.

11 Yu T, Park Y, Johnson JM, et al. apLCMS - adaptive processing of high-resolution LC/MS data. Bioinformatics 2009; 25: 1930-1936. 
12 Uppal K, Soltow QA, Strobel FH, et al. xMSanalyzer: automated pipeline for improved feature detection and downstream analysis of large-scale, non-targeted metabolomics data. BMC Bioinformatics 2013; $14: 1$.

13 Guijas C, Montenegro-Burke JR, Domingo-Almenara X, et al. METLIN: a technology platform for identifying knowns and unknowns. Anal Chem 2018; 90: 3156-3164.

14 Chapman ALP, Mocatta TJ, Shiva S, et al. Ceruloplasmin is an endogenous inhibitor of myeloperoxidase. $J$ Biol Chem 2013; 288: 6465-6477.

15 Xia J, Sinelnikov IV, Han B, et al. MetaboAnalyst 3.0 - making metabolomics more meaningful. Nucleic Acids Res 2015; 43: W251-W257.

16 Wickham H. ggplot2: Elegant Graphics for Data Analysis. 1st Edn. New York, Springer, 2009.

17 Salinas DB, Sosnay PR, Azen C, et al. Benign and deleterious cystic fibrosis transmembrane conductance regulator mutations identified by sequencing in positive cystic fibrosis newborn screen children from California. PLoS One 2016; 11: e0155624.

18 Pattison DI, Davies MJ. Absolute rate constants for the reaction of hypochlorous acid with protein side chains and peptide bonds. Chem Res Toxicol 2001; 14: 1453-1464.

19 Taylor-Cousar JL, Von Kessel KA, Young R, et al. Potential of anti-inflammatory treatment for cystic fibrosis lung disease. J Inflamm Res 2010; 3: 61-74.

20 Grasemann H, Ratjen F. Early lung disease in cystic fibrosis. Lancet Respir Med 2013; 1: 148-157.

21 Thomson E, Brennan S, Senthilmohan R, et al. Identifying peroxidases and their oxidants in the early pathology of cystic fibrosis. Free Radic Biol Med 2010; 49: 1354-1360.

22 Chandler JD, Day BJ. Biochemical mechanisms and therapeutic potential of pseudohalide thiocyanate in human health. Free Radic Res 2015; 49: 695-710.

23 Arnhold J, Monzani E, Furtmüller PG, et al. Kinetics and thermodynamics of halide and nitrite oxidation by mammalian heme peroxidases. Eur J Inorg Chem 2006; 2006: 3801-3811.

24 Skaff O, Pattison DI, Davies MJ. Hypothiocyanous acid reactivity with low-molecular-mass and protein thiols: absolute rate constants and assessment of biological relevance. Biochem J 2009; 422: 111-117.

25 Winterbourn CC. The biological chemistry of hydrogen peroxide. Methods Enzymol 2013; 528: 3-25.

26 Go Y-M, Chandler JD, Jones DP. The cysteine proteome. Free Radic Biol Med 2015; 84: 227-245.

27 Dunn WB, Erban A, Weber RJM, et al. Mass appeal: metabolite identification in mass spectrometry-focused untargeted metabolomics. Metabolomics 2013; 9: 44-66.

28 Wang B, Shi Z, Weber GF, et al. Introduction of a new critical $p$ value correction method for statistical significance analysis of metabonomics data. Anal Bioanal Chem 2013; 405: 8419-8429.

29 Zang X, Monge ME, McCarty NA, et al. Feasibility of early detection of cystic fibrosis acute pulmonary exacerbations by exhaled breath condensate metabolomics: a pilot study. J Proteome Res 2017; 16: 550-558.

30 Kettle AJ, Turner R, Gangell CL, et al. Oxidation contributes to low glutathione in the airways of children with cystic fibrosis. Eur Respir J 2014; 44: 122-129.

31 Day BJ, van Heeckeren AM, Min E, et al. Role for cystic fibrosis transmembrane conductance regulator protein in a glutathione response to bronchopulmonary Pseudomonas infection. Infect Immun 2004; 72: 2045-2051.

32 Kemp M, Go Y-M, Jones DP. Nonequilibrium thermodynamics of thiol/disulfide redox systems: a perspective on redox systems biology. Free Radic Biol Med 2008; 44: 921-937.

33 Harwood DT, Kettle AJ, Winterbourn CC. Production of glutathione sulfonamide and dehydroglutathione from GSH by myeloperoxidase-derived oxidants and detection using a novel LC-MS/MS method. Biochem J 2006; 399: $161-168$.

34 Fuschillo S, De Felice A, Balzano G. Mucosal inflammation in idiopathic bronchiectasis: cellular and molecular mechanisms. Eur Respir J 2008; 31: 396-406.

35 Polineni D, Dang H, Gallins PJ, et al. Airway mucosal host defense is key to genomic regulation of cystic fibrosis lung disease severity. Am J Respir Crit Care Med 2018; 197: 79-93.

36 Corvol H, Blackman SM, Boëlle P-Y, et al. Genome-wide association meta-analysis identifies five modifier loci of lung disease severity in cystic fibrosis. Nat Commun 2015; 6: 1-8.

37 Dang H, Gallins PJ, Pace RG, et al. Novel variation at chr11p13 associated with cystic fibrosis lung disease severity. Hum Genome Var 2016; 3: 16020-16025

38 Lee BC, Lee HM, Kim S, et al. Expression of the methionine sulfoxide reductase lost during evolution extends Drosophila lifespan in a methionine-dependent manner. Sci Rep 2018; 8: 1010

39 Lee BC, Le DT, Gladyshev VN. Mammals reduce methionine-S-sulfoxide with MsrA and are unable to reduce methionine- $R$-sulfoxide, and this function can be restored with a yeast reductase. J Biol Chem 2008; 283: 28361-28369.

40 Konstan MW, VanDevanter DR, Sawicki GS, et al. Association of high-dose ibuprofen use, lung function decline, and long-term survival in children with cystic fibrosis. Ann Am Thorac Soc 2018; 15: 485-493.

41 Nielsen VG, Webster RO. Inhibition of human polymorphonuclear leukocyte functions by ibuprofen. Immunopharmacology 1987; 13: 61-71.

42 Koelsch M, Mallak R, Graham GG, et al. Acetaminophen (paracetamol) inhibits myeloperoxidase-catalyzed oxidant production and biological damage at therapeutically achievable concentrations. Biochem Pharmacol 2010 79: 1156-1164.

43 Tidén A-K, Sjögren T, Svensson M, et al. 2-thioxanthines are mechanism-based inactivators of myeloperoxidase that block oxidative stress during inflammation. I Biol Chem 2011; 286: 37578-37589.

44 Casaril AM, Ignasiak MT, Chuang CY, et al. Selenium-containing indolyl compounds: kinetics of reaction with inflammation-associated oxidants and protective effect against oxidation of extracellular matrix proteins. Free Radic Biol Med 2017; 113: 395-405.

45 Conrad C, Lymp J, Thompson V, et al. Long-term treatment with oral $N$-acetylcysteine: affects lung function but not sputum inflammation in cystic fibrosis subjects. A phase II randomized placebo-controlled trial. J Cyst Fibros 2015; 14: 219-227

46 Griese M, Kappler M, Rietschel E, et al. Inhalation treatment with glutathione in patients with cystic fibrosis. A randomized clinical trial. Am J Respir Crit Care Med 2013; 188: 83-89.

47 Chandler JD, Min E, Huang J, et al. Antiinflammatory and antimicrobial effects of thiocyanate in a cystic fibrosis mouse model. Am J Respir Cell Mol Biol 2015; 53: 193-205. 